LA-UR-99-3290

Approved for public release; distribution is unlimited.

\section{Title: \\ X-RAY ABSORPTION SPECTROSCOPY: DIAGNOSTIC TOOL FOR PROBING MATERIAL PROPERTIES ON DYNAMIC EXPERIMENTS}

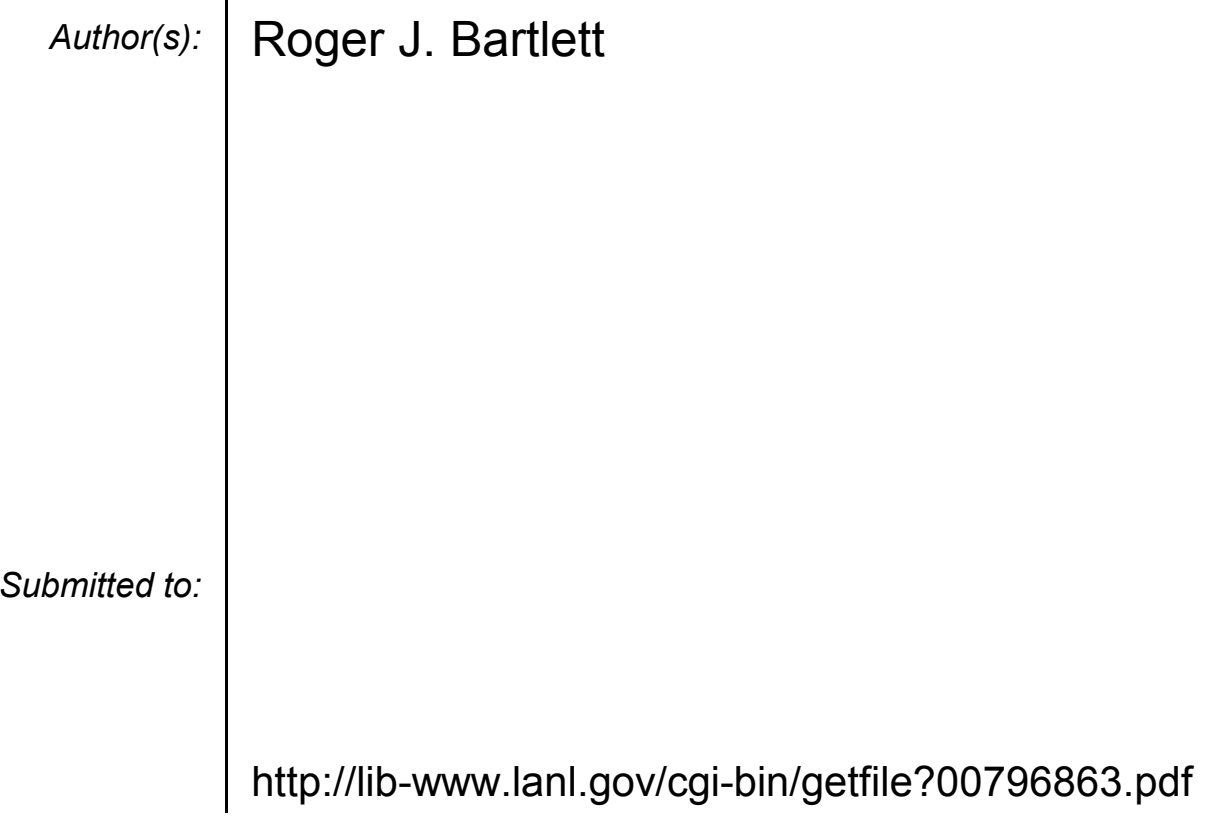




\title{
X-RAY ABSORPTION SPECTROSCOPY: DIAGNOSTIC TOOL FOR PROBING MATERIAL PROPERTIES ON DYNAMIC EXPERIMENTS*
}

\author{
Roger J. Bartlett \\ Los Alamos National Laboratory, Mail Stop D410 \\ Los Alamos, NM 87545
}

\section{Abstract}

In the most general sense we are proposing that $\mathrm{x}$-ray absorption spectroscopy (XAS) be used to extract information from laser and pulsed power experiments. The technique will not be adaptable to all types of experiments but in its many forms may be useful for a large variety of cases. Here we described a particular case in which a high $\mathrm{Z}$ (relative to the sample material) probe material is embedded in the sample and the time dependent absorption spectra of the probe is measured. The local temperature and pressure as well as other parameters affect the absorption spectrum of the probe and these parameters are then extracted from the XAS data.

\section{INTRODUCTION}

It is often necessary or desirable to "see" into the interior or to measure internal properties of a material. Often these properties are changing caused by external applied conditions or through internal reactions. This is especially true for a large class of experiments that have been designed to address stockpile stewardship issues and for code verification and validation. These experiments often deal with hydrodynamics and radiation hydrodynamics in solids, liquids and plasmas. To understand these experiments it is necessary to know the internal conditions of the sample, especially temperature and pressure. The technique proposed here is to place probe materials into the sample and to measure the x-ray absorption spectrum of the probes. The local environment will change the absorption spectrum of the probe materials and these changes can be used to measure the properties of the local environment. This approach is in some ways analogous to the use of ruby chips in diamond anvil cells to measure the pressure by observing the ruby fluorescence.

The most striking feature of $\mathrm{x}$-ray absorption is the absorption edge structure. The energy of the edge is unique for each element so it can be used to identify the element. Structure near the edge is dictated by both the electronic properties such as the empty density of states and by the structural environment of the absorbing atoms (nearest neighbors).

\footnotetext{
"Work supported by U.S. Department of Energy through
} Los Alamos National Laboratory.
As environmental factors change such as temperature and pressure both the electronic and structural properties change. These changes in turn change the absorption spectrum.

Many materials have a strong absorption peak near/at the absorption edge. The peak is caused by a large number of empty states available to the core electrons, as they are excited. This line is called a white line [1] because of its appearance on a photographic negative. Because both pressure and temperature influence the empty density of states, the white line absorption is also affected. It has been demonstrated that the temperature broadens the line and the pressure causes a shift in energy [2]. Here we will discuss these phenomena and propose their use as probes to measure the local temperature and pressure in a sample.

Pulsed power and laser facilities such as Pegasus, Nova, $\mathrm{Z}$ and later Atlas, the National Ignition Facility (NIF) and the ZX machine are being used or will be used to address Stockpile Stewardship issues and code verification and validation as well as basic and applied science issues. Each experiment is designed to address one or more issues generally in the area of hydrodynamics, radiation flow, integrated experiments or data base enhancement. Generally, the experiments use a high-energy store to drive a target/load either through magnetic compression or through a laser drive system. The experiments last only a few nanoseconds to a few microseconds. During this time, the material properties such as phase, strength, lattice constant, ionization state, etc can change significantly initiated by the tremendous amount of power/energy introduced into the sample. The challenge is to diagnose the experiment during this brief period and extract the required information. This means not only looking at surface properties of the sample but also looking into the interior. Because the samples are opaque to most radiation except $\mathrm{x}$-rays, $\mathrm{x}$-ray radiation becomes the probe of choice for views of the interior.

Radiography, images of self-emission, radiation temperature measurements, spectral analysis of the selfemission and VISAR (Velocity Interferometer System for Any Reflector) are some of the common diagnostic tools that are currently being used. Each of these techniques can give valuable information about what is happening in the interior or on the surface of the sample. However, there are also a number of limitations; e.g., radiography only gives a snapshot in time and shows the contrast through the whole sample. Self-emission is generally only 
from the exterior of the sample and does not give information about the interior regions, and VISAR only measures the shock break out (from the sample). Two parameters, temperature and pressure, if measured at known points in the sample as a function of time can shed considerable light on the sample properties; e.g., the passage of shock waves. (Shock waves are singled out because they play a very important role in many high energy density experiments.) A method for determining the time evolution of these parameters will be described here. The diagnostic proposed here is not meant to replace the more common diagnostics but to supplement them and add further value to the experiments.

\section{APPROACH}

X-ray absorption spectroscopy (XAS) as the name implies measures the energy resolved attenuation of the $\mathrm{X}$ ray beam through a sample. Information about the electronic and structural properties of the material can be distilled from the measured absorption coefficient. Although this information may be valuable, it generally does not come from a localized point in the sample. However, because each element has a characteristic absorption structure (absorption edges) each material can be identified and if a particular material is localized in the sample then position sensitive information can be measured. This is the essence of the proposed measurements; i.e., to measure the absorption properties of localized "probe materials" to obtain position sensitive information about the sample. It remains to be demonstrated that the absorption spectrum of the probe materials can be measured and that the probe materials' environment changes the spectrum in a measurable manner.

X-ray absorption spectra are generally decreasing functions of photon energy punctuated by abrupt increases at absorption edges: energies just sufficient to excite core electrons to the first available empty states. For somewhat higher energies, the core electrons are ionized. Often the spectrum around the edges is divided into regions that depend on different aspects of the electronic and structural properties of the material. The region near the edge where the density of states/electronic properties and nearest neighbor atoms are important in determining the cross section is called $\mathrm{x}$-ray absorption near edge structure (XANES) [3]. For energies grater than several tens of eV above the edge, small variations in the absorption structure occur caused by interference effects of the photoelectron wave scattering off surrounding atoms. This structure is called extended $\mathrm{x}$-ray absorption fine structure (EXAFS) [4]

The environment of the absorbing atom influences each of these regions of the spectrum. Often the resulting change in the spectrum caused by changes in the environment is small but measurable under optimum conditions. The goal here is to determine the environmental conditions from the measured spectrum. Each of these regions of the spectrum can provide valuable information about the environment of the probe atom and thus about the sample itself. However, based on known cross sections, the near-edge structure will give the best signal to noise ratio particularly in those elements that have a white line. The probe elements with white lines will be chosen so that the $\mathrm{x}$-ray edge is at a sufficiently high energy so that the $\mathrm{x}$-rays will penetrate the sample (often a low $\mathrm{Z}$ material).

\section{EXPERIMENT}

The experiment can be broken down into three main components. These are the x-ray source including the curved crystal monochromator, the sample including the probe materials and the data acquisition system including the time resolved two-dimensional detector. A schematic layout of this generalized set up is shown in Figure 1.

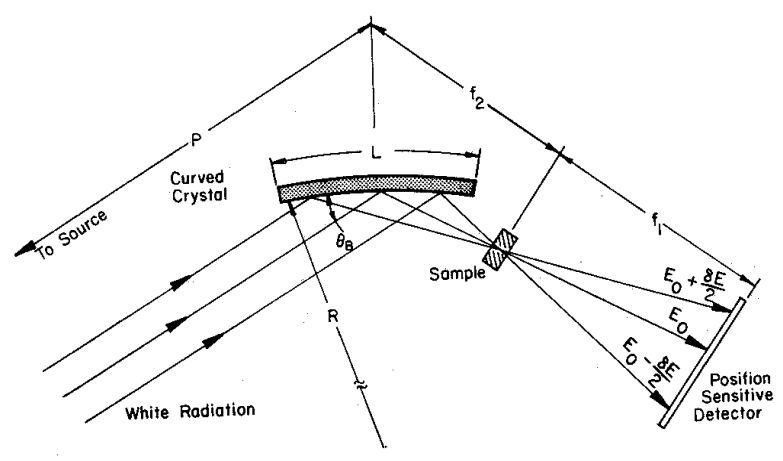

Fig. 1. Schematic of energy dispersive absorption measurement.

\section{A. X-ray Source}

If the experiment is designed to look only at the absorption edge and near edge structure, then the x-ray source needs to be continuous only over this relatively narrow range. Generally, 50 to $100 \mathrm{eV}$ will be sufficient. The source also has to have the proper time structure. Generally, the source will be pulsed. The pulse will either be short relative to the duration of the experiment, or the duration of the x-ray pulse will be on the order of or longer than the duration of the experiment. If the pulse is short, the source will determine the timing and time resolution of the snap shot. Multiple snap shots will require multiple $\mathrm{x}$-ray pulses from one or more sources. If the pulse is long, the timing and time resolution will be determined by the detector system (discussed later). 
There are several types of pulsed $\mathrm{x}$-ray generators that produce broad spectral radiation. These include high voltage pulsed sources, accelerator based sources and laser plasma sources. The spectrum usually consists of a broad Bremsstrahlung continuum and some characteristic line radiation. The Bremsstrahlung radiation can be modified somewhat by adjusting the electron acceleration. These sources can provide pulse duration of several nanoseconds to several microseconds. The main disadvantage of these sources is the limited intensity in the range of interest. This is partly caused by the fact that the $x$-ray energy is emitted into a large solid angle and only a small fraction is directed at the sample. In addition, the spectrum is spread over a very wide energy range while only a relatively narrow band of radiation is needed.

The laser driven sources can produce radiation in the $1 \mathrm{keV}$ to $5 \mathrm{MeV}$ energy range. This is enabled by the high irradiance lasers $\left(\sim 10^{21} \mathrm{~W} / \mathrm{cm}^{2}\right)$ producing hot electrons which in turn produce conventional Bremsstrahlung radiation. The radiation spectrum can be adjusted somewhat by adjusting the irradiance. The x-ray radiation has essentially the same time structure as the laser drive.

Potentially a superior source is a parametric $\mathrm{x}$-ray (PXR) source [5]. In this case, an electron (any charged particle) passing through a medium with periodic varying permittivity (dielectric constant), such as a crystal produces radiation. The radiation is produced in narrow spectral bands and is collimated. The process is relatively new in that it was predicted in 1957 and first observed in 1985. Further investigation and work on this type of source may prove useful.

\section{B. Monochromator}

In order to produce an absorption spectrum, the broad band radiation needs to be monochromated. In addition, the complete spectrum needs to be recorded simultaneously. This can be accomplished by using a curved crystal as shown in Figure 1. The broad band radiation strikes the crystal at slightly different angles across the face; thus spreading the energy in position on a one or two-dimensional detector. The radiation also forms a line focus at the sample. It is also possible to use a bent crystal in a transmission mode [6], which may have some advantages at higher photon energies.

\section{Probe Materials}

The embedded probe materials need to have an x-ray absorption edge with a white line structure in an energy range where the sample is fairly transparent to the radiation. Generally, this means that the probe material has a much higher $Z$ than the sample and that the $\mathrm{K}$ edge absorption of the probe is being measured. Unfortunately, most metals do not have a white line associated with their K edge absorption structure. This is because the dipole selection rules force the core $\mathrm{s}$ electrons to couple with $\mathrm{p}$ symmetric band states, which are usually broad and do not have a high density of states. Often this can be overcome by using the oxide of the metal. The oxides often have exciton states near the x-ray edge that have $p$ like symmetry that can give rise to white line absorption.

The probe material can be embedded in the sample in the form of clusters, pellets, wires/cylinders or most any shape that can be manufactured. To illustrate the technique, a wire probe in a cylindrical sample is shown in Figure 2. In this case the wire is on a diameter of the sample and is placed at the line focus of the radiation (see Figure 3 below).

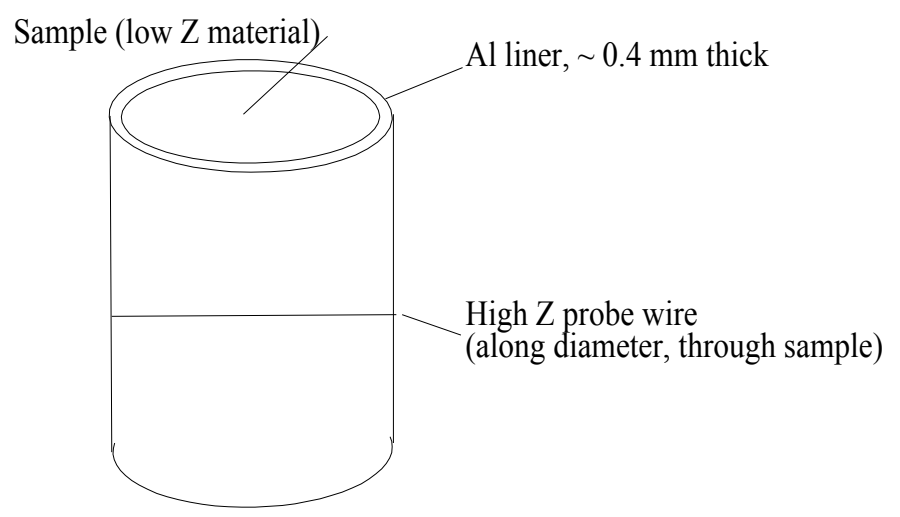

Fig. 2. Schematic of pulse power load showing Aluminum liner, low $\mathrm{Z}$ material (sample) and high $\mathrm{Z}$ probe along sample diameter.

By measuring the $x$-ray absorption along the probe wire, it will be possible to determine the temperature and pressure along the diameter of the sample. This will also give the velocity and intensity of shock waves converging toward the center of the sample and reflecting back along the diameter.

\section{Detector System}

The detector requirements are a function of the type of experiment and the data requirements. Figure 3 illustrates the potential for obtaining an x-ray absorption spectrum as a function of position in the sample and as a function of time. Whether all or just some of these data are recorded will depend on the detector system. The figure shows the $\mathrm{x}$-ray beam after it has been Bragg reflected from the curved crystal. The beam is line focussed at the probe material and the energy is dispersed as shown. It can be seen that a two-dimensional detector is required to record the complete energy spectrum as a function of position along the probe. In its simplest form, 
this can be photographic $\mathrm{x}$-ray film or an image plate. Two-dimensional CCD arrays with the appropriate $\mathrm{x}$-ray converters can also be used. Because these detectors do not provide time-resolved data, The time resolution is obtained from the pulsed $\mathrm{x}$-ray source. Essentially only one snap shot can be recorded under this configuration. However, if the sample is symmetric relative to a plane through the axis then it may be possible to record two spectra using a double pulse x-ray source.

Using a time resolved detector and a multi pulsed (or multi unit) x-ray source, several time frame can be recorded on one shot. Alternatively, using a time resolved detector and an $\mathrm{x}$-ray source that is on for the duration of the experiment can provide multi snap shots or a continuous record of the absorption spectrum. A steak camera can be used to record continuously but because it gives only one dimension, it will record the spectrum at only one location along the probe. On the other hand, a framing camera will produce several two dimensional snap shots. This may be the optimum because it will give both time and position resolved information.

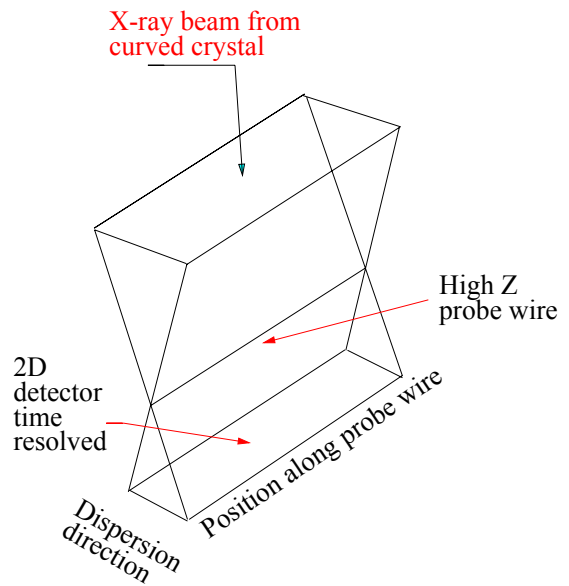

Fig. 3. Schematic of x-ray beam from the curved crystal monochromator showing the line focus at the probe position in the sample. In this illustration, the absorption spectrum as a function of position along the probe wire can be recorded.

\section{CONCLUSION}

We have proposed a technique for determining the temperature and pressure as a function of time at specific locations (location of probe materials) in samples undergoing extreme physical change. The technique is based on the fact that the x-ray absorption spectrum of a material is changed in a measurable way by the local temperature and pressure. Rough estimates of the feasibility of the technique based on existing sources and detectors suggest that the measurement will be difficult but under optimum conditions should be feasible.
X-ray source development and detector development for other programs such as radiography will also benefit the measurements proposed here.

\section{REFERENCES}

1. M. Brown, R. E. Peierls and E. A. Stern, "White lines in X-ray absorption," Phys. Rev. B, vol. 15, 738-744, Jan. 1977.

2. G. Faraci, A. R. Pennisi, and J. L. Hazemann, "XANES of high-pressure Kr clusters in Be and Si," Phys. Rev. B, vol. 56, pp. 12553-12559, Nov. 1997.

3. A. Bianconi, "XANES Spectroscopy" in X-ray absorption : principles, applications, techniques of EXAFS, SEXAFS, and XANES, D.C. Koningsberger and R. Prins, ed. New York: Wiley, 1988, pp. 573662.

4. E. A. Stern and S. M. Heald, "Basic principles and applications of EXAFS," in Handbook on Synchrotron Radiation, Vol. 1B, Ernst-Eckhard Koch, Ed. Amsterdam, New York, Oxford: NorthHolland Publishing Co., 1983, pp. 955-1014.

5. A. V. Shchagin, V. I. Pristupa and N.A. Khizhnyak, "A fine sturcture of parametric X-ray radiation from relativistic electrons in a crystal," Physics Letters A, vol. 148, pp. 485-487, Sept. 1990.

6. Z. Zong, D. Chapman, W. Thomlinson, F. Arfelli and R. Menk, "A bent Laue crystal monochromator for momochromatic radiography with an area beam," Nucl. Instr. Meth. in Phys. Res. A, vol. 399, pp. 489498, 1997. 